\title{
Novel targets in old rogues: Integrative structural biology approach for discovery of natural product inhibitors
}

\author{
M. Antil, S. Gupta, D. Verma, J. Mathur, V. Gupta \\ Biotechnology Department, Jaypee Institute of Information Technology (JIIT), A-10, sector-62, Noida, 201309, India \\ vibha.gupta@jiit.c.in
}

Prevalence of drug-resistant strains of causative agents of age old diseases pneumonia and tuberculosis (TB), has urged focus on exploring novel targets and development of new therapeutics with a fresh perspective in the battle against antibiotic resistance. Nowa-days bioactive compounds from natural origin are superseding the use of synthetic compounds due to structural and chemical diversity [1]. Our research illustrates the power of integrative structural biology in the discovery of inhibitors against two potential drug targets - (1) Serine acetyltransferase (also known as CysE), an enzyme of de novo cysteine biosynthetic pathway, and (2) Isocitrate lyases with role in both glyoxylate cycle and methylcitrate cycle

(1) CysE catalyzes the production of O-acetyl-L-serine (OAS) from acetyl-CoA and L-serine. The enzyme, essential for survival in a mouse model of TB infection [2], is absent in Homo sapiens. Therefore, this target is worth exploring for developing new antimicrobial compounds. The crystal structure of K. pneumoniae (Kpn) CysE was solved and used as a receptor for blind docking of natural compounds with documented antioxidant, antibacterial, respiratory stimulant, anti-inflammatory, and bronco-dilatory activities. L-Cys, a feedback inhibitor of CysE which binds at the active site was also docked as a positive control (Fig.1a). The best binders were tested for the inhibitory potential of CysE and quercetin was identified as the most potent inhibitor (Fig. 1b). MD simulations verified it as an allosteric inhibitor that binds at the trimer-trimer interface distal to the active and cofactor binding site.

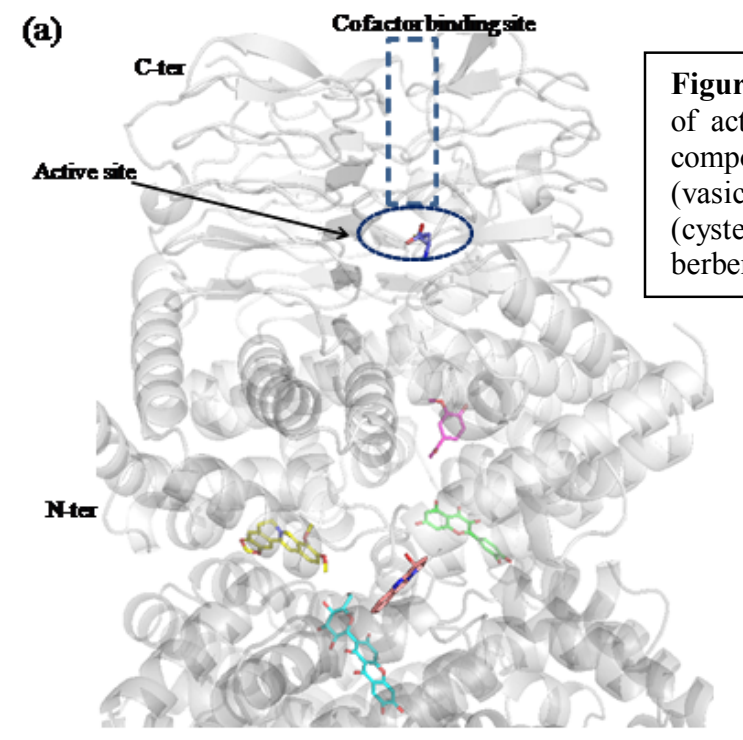

Figure 1(a). Docking of natural compounds within Kpn CysE structure. Location of active site (dotted oval), cofactor binding site (dotted rectangle) and natural compounds in stick representation are depicted in magenta (apocynin), peach (vasicine), cyan (mangiferin), yellow (berberine), green (quercetin) and blue (cysteine) colors. (b). \% inhibition of Kpn CysE by natural compounds - apocynin, berberin, mangiferin, quercetin and vasicine along with known inhibitor L-cysteine.

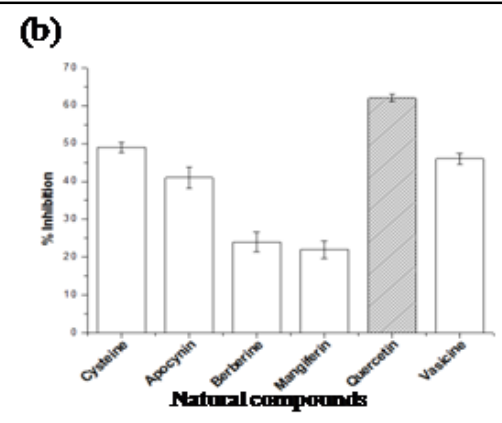

(2) Isocitrate lyases (ICL1/ICL2) are essential for persistence of M. tuberculosis (Mtb) in its host [3] as they play an important role in metabolism of even and odd chain fatty acids via $\beta$-oxidation. Though high resolution crystal structures of Mtb ICL1 are available in PDB since 2000, and GlaxoSmithKline-TB Alliance launched high throughput screening of 900,000 compounds to identify ICL1 inhibitor, their efforts culminated in modest succes, in view of poor characterization of ICL2 structure-function relationship. We purified both Rv1915 and Rv1916 and characterized them possessing dual isocitrate and methylisocitrate lyase activities akin to ICL1[4]. In silico screening of natural compounds has yielded an inhibitor which is able to abolish both the activities in all Mtb ICLs.

[1]. Pereira D. M., Andrade C., Valentão P., \& Andrade P. B. (2017). “Natural Products Targeting Clinically Relevant Enzymes, pp. 1-18. WileyVCH Verlag GmbH \& Co. KGaA,

[2]. Sassetti C. M. \& Rubin E. J. (2003).Proc. Natl. Acad. Sci. USA 100:12989-94

[3]. McKinney J. D., zu Bentrup K. H., Muñoz-Elías E. J., et al (2000). Nature 406:735-738.

[4]. Gould T. A., van de Langemheen H., Munoz-Elias E. J. , et al (2006). Mol Microbiol 61:940-947.

Keywords: Serine acetyltransferase, Isocitrate lyase, novel targets, natural inhibitors

Acta Cryst. (2021), A77, C99 